\title{
Material Optimization of Connecting Rod Using Finite Element Analysis
}

\author{
Anjan Gautam ${ }^{1}$, Er. Gurmeet Singh ${ }^{2}$ \\ Department of Mechanical Engineering, P.C.E.T Lalru, Punjab (India)
}

\begin{abstract}
Connecting rod is a major part inside the engine as it connects the piston to the crankshaft and also used to transfer power from the piston to the crankshaft. Connecting rods of vehicles are generally manufactured by the process of forging or casting (Either wrought steel or powdered metal). Under complex loading connecting rod of an automobile vehicle a high production component. The weight and design of the connecting rod have a good influence on performance of an automobile engine. Use of lighter material can increase the performance of an engine because of its less energy consumption than heavier material. Conventional materials have limitations in strength, stiffness and toughness, and because of this they have failed to fulfil the demand of saving of energy. This shows the need to work upon materials having high strength and less weight as compared to conventional materials. Use of composite material would help to reduce weight and improve fuel consumption without sacrificing the safety of the vehicle. In this current work, connecting rod has been designed by using computer aided design (CAD) software CATIA and was analysed using a finite element analysis (FEA) software ANSYS to understand the exact behaviour of the connecting rod under different material properties.
\end{abstract}

Keywords: Connecting Rod, ANSYS, FEA, CATIA

\section{Introduction}

A connecting rod is an engine component which transfers motion from the piston to the crankshaft.It functions as a lever arm. It converts reciprocating motion into rotating motion. As connecting rod is rigid, and it may transmit either a push or a pull. Earlier mechanisms, such as chains, could only pull. In a few two-stroke engines, the connecting rod is only required to push.

A combination of axial and bending stresses act on the rod in operation. The axial stresses produced arethe product of cylinder gas pressure and the inertia force, which arising on account of reciprocating motion. Whereas bending stresses produce due to the centrifugal effects.

A typical connecting rod consist of a pin -end, a shank section, and crank end. A pin end and crank end pin holes are machined to permit accurate fitting of bearings. One end of the connecting rod is connected to the piston by the piston pin. The classification of connecting rod is made by the cross sectional point of view i.e. I - section, $\mathrm{H}$ - section, Tabular section, Circular section.

Kuldeep et al. (2013) carried out FEA analysis by considering two materials. Various parameters were obtained from ANSYS software like von misses stress, von misses strain and displacement. In the results when the analysis was compared to the former material the new material found to had less weight and better stiffness. It resulted in reduction of $43.48 \%$ of weight, with $75 \%$ reduction in displacement.

Ramani et al (2014) carried out a study in which the main idea was to do analysis of connecting rod and get idea of stress producing during compressive and tensile loading. In the results they give idea about weight reduction opportunities for a production steel connecting rod. The study had two subjects, first, load and stress analysis of the connecting rod, and second, optimization for weight reduction. The first part of the study consists of, loads acting on the connecting rod and find out stress-time history at some critical point. The results were also used to determine the variation of Tensile and Compressive loading the component was optimized for weight reduction subject to space constraints and manufacturability.

Tiwari et al (2014) took the phenomenon that fatigue should be taken into account during the development, in order to guarantee the connecting rod required lifetime. Numerical tools had been extremely used during the connecting rod development phase. All the work showed the complete connecting rod Finite Element Analysis (FEA) methodology to explore weight and cost reduction opportunities for a production of forged steel connecting rod. It was also performed a fatigue study based on Stress Life (SxN) theory, considering the Modified Goodman diagram.

Savanoor et al (2014) compared the von mises stress and total deformation of 2 different aluminium alloys with the forged steel. FEA analysis was carried out by considering three materials. The parameters like von misses stress and displacement were obtained from ANSYS software. Then compared the aluminium alloys with the forged steel. In the results Al5083 alloy found to have less weight with reduction of $63.19 \%$ of weight.

\section{Development of Geometry}

According to Rankine formulae:-

$$
\mathrm{W}_{\mathrm{cr}} \text { about } \mathrm{X} \text { axis }=\frac{[\sigma c \times A]}{1+a\left[\frac{L}{K x x}\right]^{2}}=\frac{[\sigma c \times A]}{1+a\left[\frac{l}{K x x}\right]^{2}}
$$

$[\therefore$ forbothendshinged $L=l]$ 


\section{International Journal of Science and Research (IJSR) \\ ISSN (Online): 2319-7064}

Index Copernicus Value (2013): 6.14 | Impact Factor (2015): 6.391

$\mathrm{W}_{\mathrm{cr}}$ about $\mathrm{Y}$ axis $=\frac{[\sigma c \times A]}{1+a\left[\frac{L}{K y y}\right]^{2}}=\frac{[\sigma c \times A]}{1+a\left[\frac{l}{2 K y y}\right]^{2}}$

$\left[\because\right.$ forbothends fixed $\left.L=\frac{l}{2}\right]$

In order to have a connecting rod equally strong in buckling about both the axis, the buckling loads must be equal. i.e.

$$
\begin{aligned}
& \frac{[\sigma c \times A]}{1+a\left[\frac{l}{K x x}\right]^{2}}=\frac{[\sigma c \times A]}{1+a\left[\frac{l}{2 K y y}\right]^{2}} \text { Or, }\left[\frac{l}{K x x}\right]^{2}=\left[\frac{l}{2 K y y}\right]^{2} \\
& K^{2} x x=4 K^{2} \text { yy Or, Ixx=4Iyy }\left[\because I=A \times K^{2}\right]
\end{aligned}
$$

This shows that the connecting rod is four times strong in buckling about $\mathrm{x}$-axis than about- $\mathrm{y}$-axis. If I xx $>$ 4Iyy, Then buckling will occur about $\mathrm{y}$-axis and if I xx < 4Iyy, then buckling will occur about x-axis .In Actual practice I $\mathrm{xx}$ is kept slightly less than 4Iyy. It is usually taken between 3 and 3.5 and the Connecting rod is designed for buckling about $\mathrm{x}$-axis. The design will always be satisfactory for buckling about y-axis. The most suitable section for the connecting rod is I-section with the proportions shown in fig. 4.1.

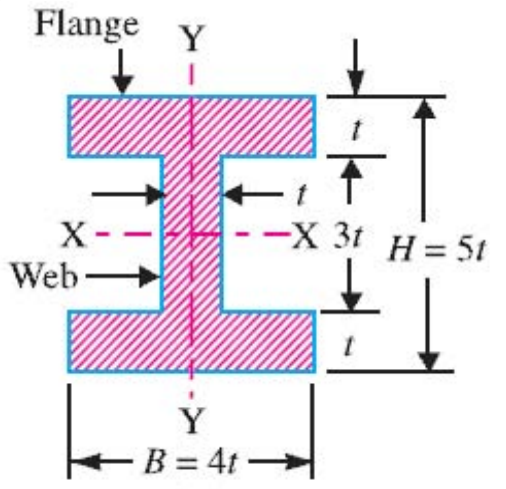

(a)

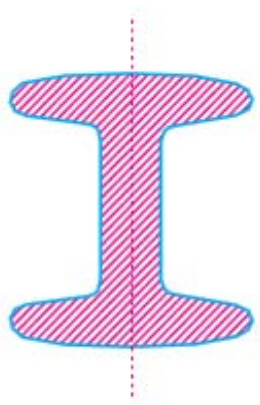

(b)

Area of the cross section $=2[4 t \times t]+3 t \times t=11 t^{2}$

Moment of inertia about $\mathrm{x}$-axis $=\frac{1}{12}\left(B D^{3}-b d^{3}\right)$

$=\frac{1}{12}\left(4 t(5 t)^{3}-3 t(3 t)^{3}\right)=\frac{419}{12}\left(t^{4}\right)$

Moment of inertia about y-axis =

Iyy $=\frac{2 \times 1}{12} \times t \times(4 t)^{3}+\frac{1}{12}(3 t) t^{3}=\frac{131}{12}\left(t^{4}\right)$

$\therefore \frac{\text { Ixx }}{\text { Iyy }}=\frac{419}{12} \times \frac{12}{131}=3.2$

Since the value of $\frac{I x x}{\text { Iyy }}$ lies between 3 and 3.5, therefore Isection chosen is quite satisfactory.

Table 1.1: Engine Specification taken into Consideration

\begin{tabular}{|c|c|}
\hline Company & Bajaj pulsar 220 \\
\hline Engine type & Air cooled 4 stroke \\
\hline Bore X Stroke(mm) & $67 \times 62.4$ \\
\hline Displacement & $220 \mathrm{CC}$ \\
\hline Maximum power & 21.05 \\
bhp@8500rpm \\
\hline Maximum torque & 19.12 \\
\hline Compression ratio & $9.35: 1$ \\
\hline Explosion pressure & $4.1 \mathrm{Mpa}$ \\
\hline Weight of piston assembly & $0.3 \mathrm{Kg}$ \\
\hline Working temperature & $-30^{0} \mathrm{C}$ to $180{ }^{\circ} \mathrm{C}$ \\
\hline
\end{tabular}

\section{Materials and Methods}

Table 1.2: Material Properties Selected

\begin{tabular}{|c|c|c|c|}
\hline Properties & Al 10\% SiC & Al 20\% SiC & Al 30\% SiC \\
\hline Density $\left(\mathrm{Kg} \mathrm{m}^{-3}\right)$ & 2742 & 2784 & 2826 \\
\hline Young's modulus ( MPa) & 93010 & $1.0901 \mathrm{E}+05$ & $1.1721 \mathrm{E}+05$ \\
\hline Poisson ratio & 0.295 & 0.29 & 0.3 \\
\hline Coefficient of thermal expansion $\left(\mathrm{C}^{-1}\right)$ & 21.04 & 14.994 & 12.4 \\
\hline Tensile Strength $(\mathrm{MPa})$ & 460 & 572 & 589 \\
\hline Compressive strength $(\mathrm{MPa})$ & 350 & 430 & 460 \\
\hline Thermal Conductivity $\left(\mathrm{Wm}^{-1} \mathrm{~K}^{-1}\right)$ & 92.993 & 124 & 124 \\
\hline Specific heat $\left(\mathrm{Jkg}^{-1} \mathrm{~K}^{-1}\right)$ & 929.89 & 914 & 800 \\
\hline
\end{tabular}

\begin{tabular}{|c|c|}
\hline Properties & Forged Steel \\
\hline Density (g/cc) & 7.7 \\
\hline Average hardness (HRB) & 101 \\
\hline Modulus of elasticity (Gpa) & 221 \\
\hline Yield strength (Mpa) & 625 \\
\hline Ultimate strength (Mpa) & 625 \\
\hline Poison ratio & 0.29 \\
\hline
\end{tabular}




\section{International Journal of Science and Research (IJSR) \\ ISSN (Online): 2319-7064}

Index Copernicus Value (2013): 6.14 | Impact Factor (2015): 6.391

\section{Design Calculations}

Calculation of buckling load

Buckling load, $\mathrm{W}_{\mathrm{b}}=$ maximum gas force $\mathrm{x}$ factor of safety

$$
=\frac{3.14 \times \mathrm{D}^{2}}{4} \times \mathrm{P} \times \text { fos }=\frac{3.14 \times\left(67 \times 10^{-3}\right)^{2}}{4} \times 4.1 \times 10^{6} \times 6=86687.079 \mathrm{~N}
$$

\section{Section profile calculations}

$$
\mathrm{W}_{\mathrm{b}}=\frac{\left(\sigma_{\mathrm{c}} \times \mathrm{A}\right)}{1+\mathrm{a}\left(\frac{\mathrm{L}}{\mathrm{Kxx}}\right)^{2}}
$$

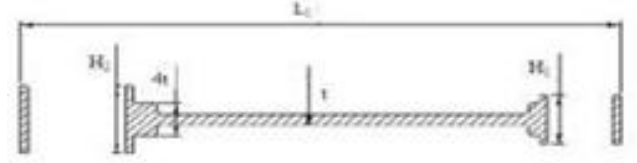

Where, $\mathrm{W}_{\mathrm{b}}=$ Buckling load, $\mathrm{A}=$ area of $\mathrm{I}$ section $=11 \mathrm{t}^{2}$

$\mathrm{L}=$ Length of connecting rod $=2 \mathrm{x}$ stroke length $=2 \mathrm{x} 62.4=$ $125 \mathrm{~mm}$

$K x x=\frac{I x x}{A}=1.78 t$,

$a=$ constant for material $=\frac{\sigma_{\mathrm{c}}}{\pi^{2} \times \mathrm{E}}, \sigma_{\mathrm{c}}=$ compressive

yield strength

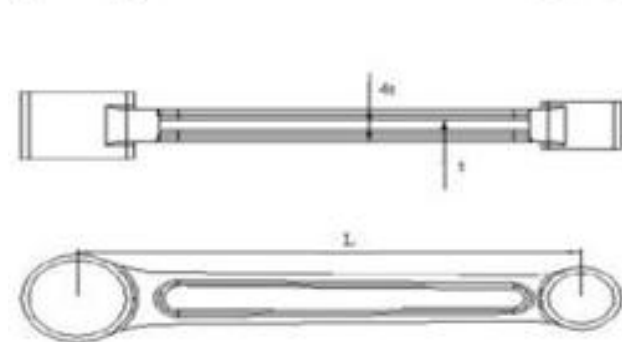

By substituting value of $\sigma_{c}, W_{b}, a, K_{x x}, A$ and $L$ we can calculate (thickness of profile)

Figure 1.2: Dimensions of Connecting Rod

\begin{tabular}{|c|c|c|c|c|c|}
\hline Dimensions & $\mathrm{t}$ & B & $\mathrm{H}$ & $\mathrm{H}_{2}$ & $\mathrm{H}_{1}$ \\
\hline Material & $(\mathrm{mm})$ & $(\mathrm{mm})$ & $(\mathrm{mm})$ & $(\mathrm{mm})$ & $(\mathrm{mm})$ \\
\hline $\mathrm{Al}+10 \% \mathrm{SiC}$ & 5 & 20 & 25 & 27.5 & 22.5 \\
\hline $\mathrm{Al}+20 \% \mathrm{SiC}$ & 4.5 & 18 & 22.5 & 24.8 & 20.3 \\
\hline $\mathrm{Al}+30 \% \mathrm{SiC}$ & 4.3 & 17.2 & 21.5 & 23.7 & 19.4 \\
\hline Forged Steel & 3.3 & 13.2 & 16.5 & 18.2 & 14.9 \\
\hline
\end{tabular}

Table 1.3: Dimensions obtained From Design Calculations

Working force on the connecting rod

$$
\mathrm{F}_{\mathrm{m}}=\mathrm{F}_{\mathrm{p}}+\mathrm{F}_{\mathrm{i}}
$$

Where, $F_{m}=$ Maximum force, $F_{p}=$ Pressure force, $F_{i}=$ Inertia force

Now, $\mathrm{F}_{\mathrm{p}}=$ Area of piston head $\mathrm{x}$ Explosion pressure

$=$

$$
\begin{aligned}
& \frac{3.14 \times \mathrm{D}^{2}}{4} \times \mathrm{P} \times=\frac{3.14 \times(67 \times 1}{4} \\
& \left\{\begin{array}{l}
\mathrm{D}=67 \mathrm{~mm} \\
P=4.1 \mathrm{MPa}
\end{array}\right\} \\
& F_{i}(\max )=\mathrm{m}_{\mathrm{r}} \times \omega^{2} \times \mathrm{r}\left(1+\frac{1}{\mathrm{n}}\right)
\end{aligned}
$$

Where, $\mathrm{m}_{\mathrm{r}}=$ mass of reciprocating parts

$=$ mass of piston assembly $+\frac{1}{3}$ rd mass of connecting rod

Mass of piston assembly $=0.3 \mathrm{~kg}$ $\omega=$ Angular velocity of crank = $\frac{2 \Pi \mathrm{N}}{60}=\frac{2 \times 3.14 \times 8500}{60}=889 \mathrm{rad} / \mathrm{sec}$

$\mathrm{r}=$ radius of crank $\frac{\text { Stroke of piston }}{=14448 \mathrm{~N}}=\frac{62.4}{2}=31.2 \mathrm{~mm}$

$\mathrm{n}=$ ratio of length of connecting rod = $\frac{\text { length of connecting rod }}{\text { radius of crank }}=\frac{125}{31.2}=4.006$

Table. 1.4: Forces Obtained from Design Calculations

\begin{tabular}{|l|c|c|}
\hline \multicolumn{2}{|c|}{ Material } & Total force (Fp +Fi) (N) \\
\hline \multicolumn{2}{|c|}{ Forged Steel } & 26055 \\
\hline concept 1 & $\mathrm{Al}+10 \% \mathrm{SiC}$ & 25171 \\
\hline concept 2 & $\mathrm{Al}+20 \% \mathrm{SiC}$ & 24976 \\
\hline concept 3 & $\mathrm{Al}+30 \% \mathrm{SiC}$ & 24904 \\
\hline
\end{tabular}

Modelling \& Analysis 
International Journal of Science and Research (IJSR)

ISSN (Online): 2319-7064

Index Copernicus Value (2013): 6.14 | Impact Factor (2015): 6.391
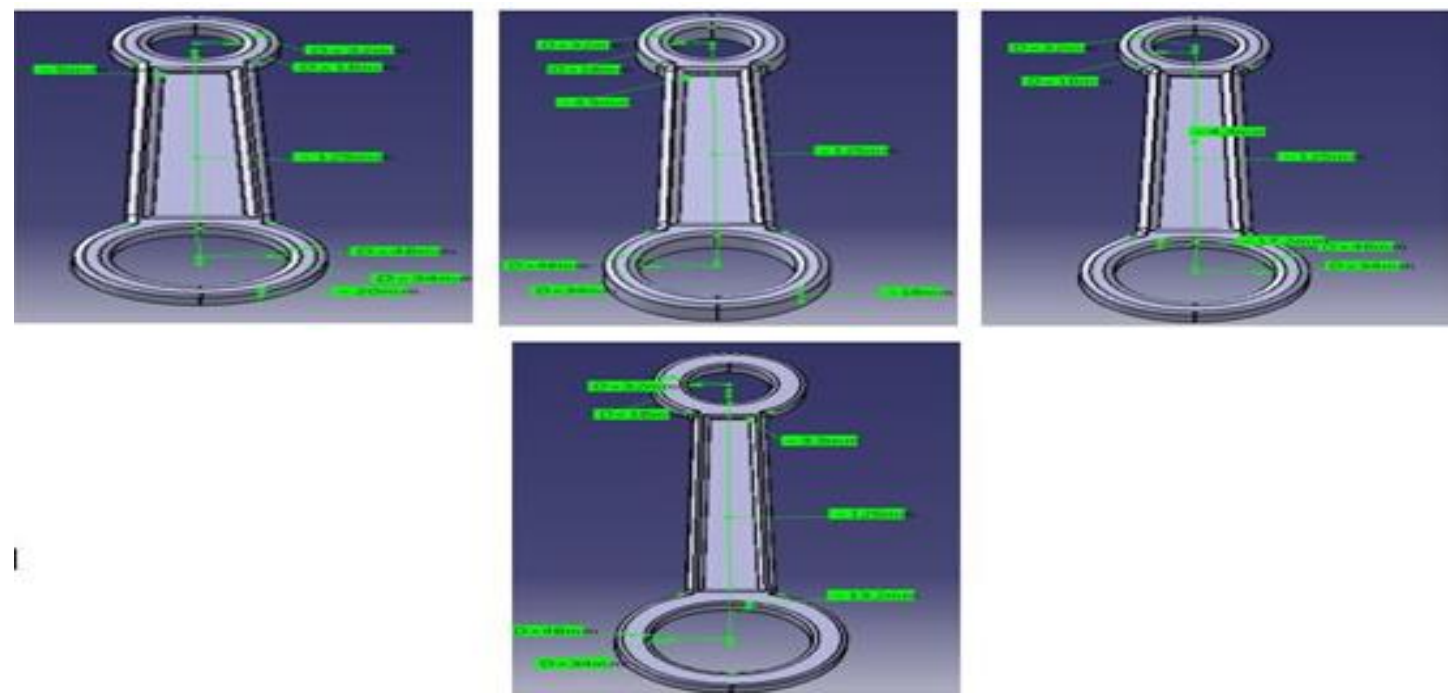

Figure 1.3: Models of Connecting Rod according to Design Calculations

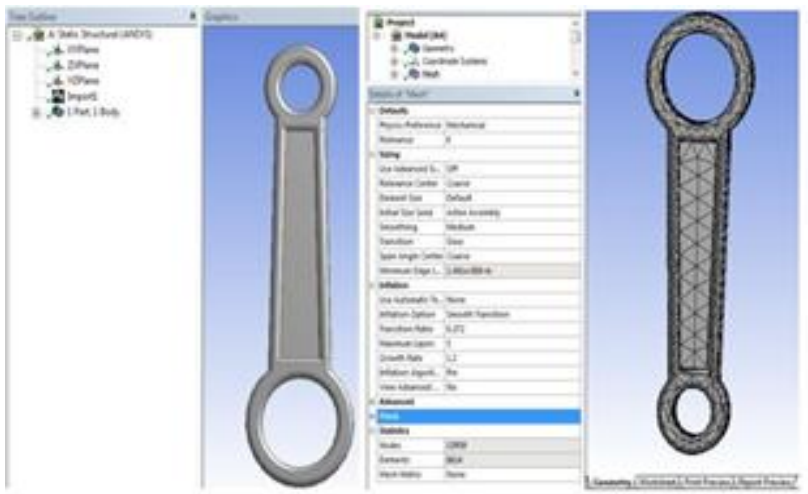

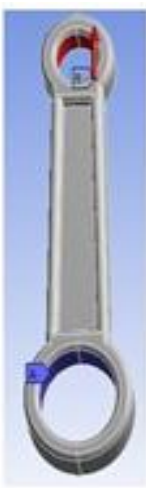

(Case 1)

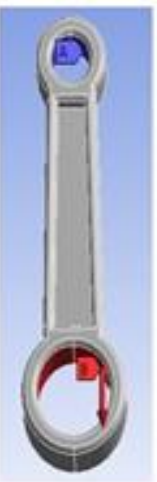

(Case 2)

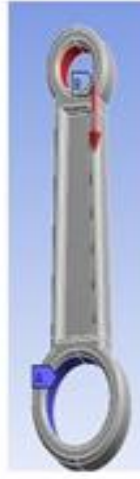

(Case 3)

Figure 1.4: Meshed Model \& Different Loading Cases Taken into Consideration

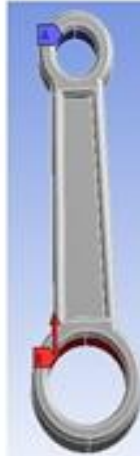

(Case 4)
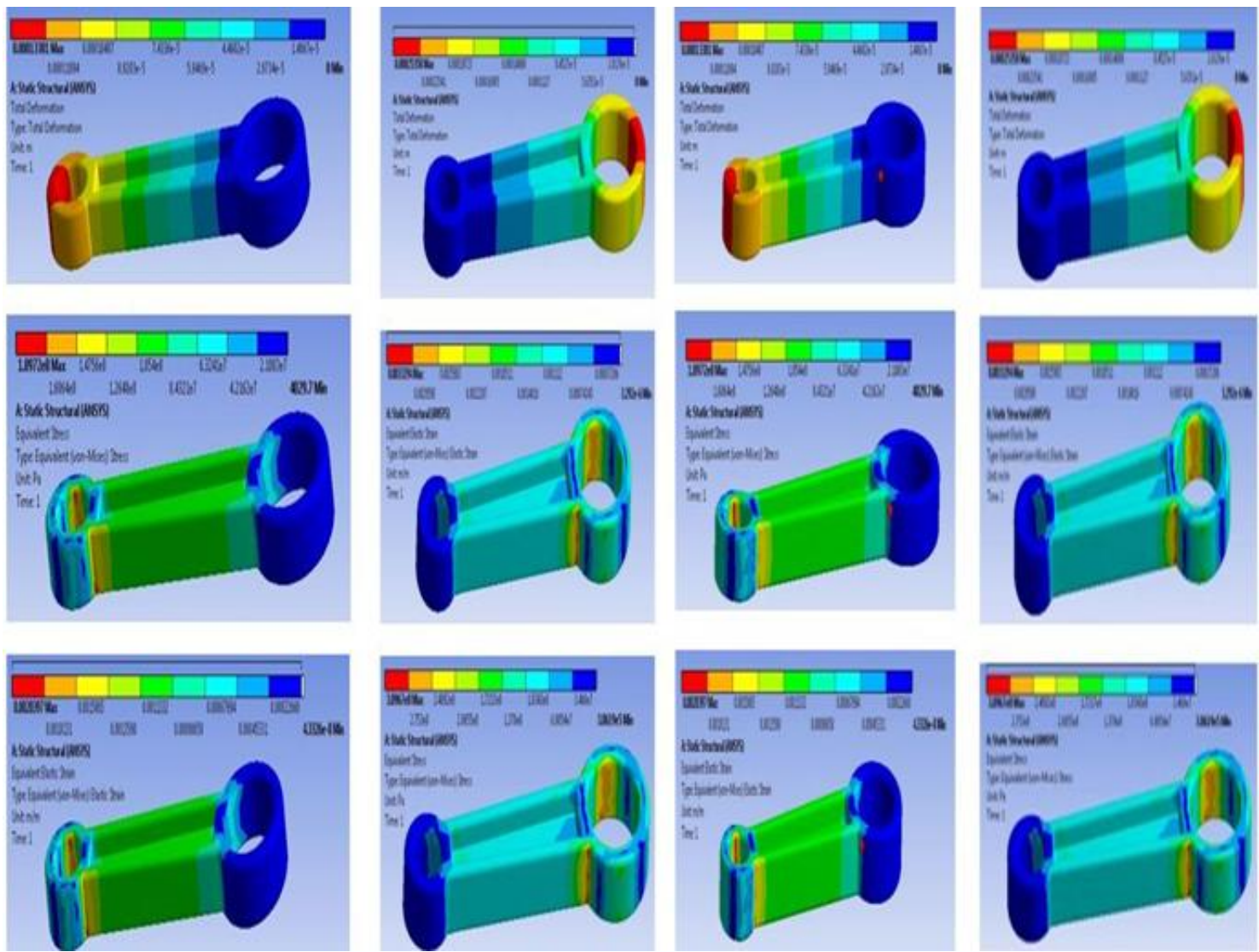

Volume 5 Issue 4, April 2016 www.ijsr.net 


\section{International Journal of Science and Research (IJSR) \\ ISSN (Online): 2319-7064}

Index Copernicus Value (2013): 6.14 | Impact Factor (2015): 6.391
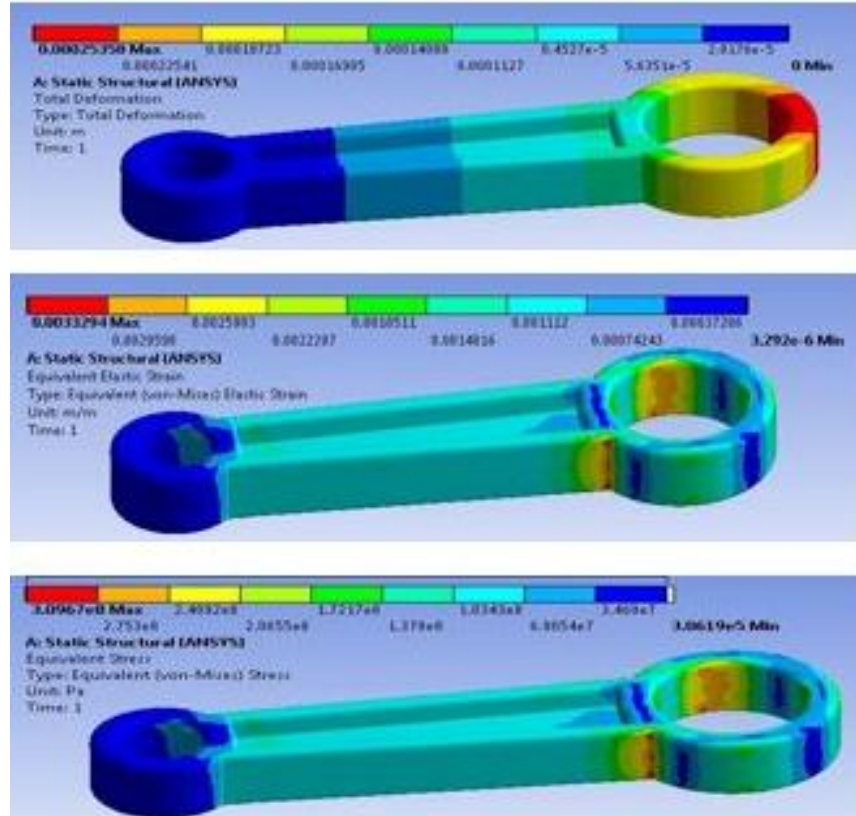

Figure 1.5: Total deformation, Equivalent Stress \& Equivalent Strain obtained for Al $+10 \%$ SiCunderLoading Case 1, Case 2, Case 3 and Case 4

Table 1.5: Combined Analysis Report for $\mathrm{Al}+10 \% \mathrm{SiC}$ with all Loading Cases

\begin{tabular}{|c|c|c|c|}
\hline & Maximum stress (MPa) & Maximum Strain & Total deformation $(\mathrm{m})$ \\
\hline Case 1 & 189.72 & 0.0020397 & 0.00013381 \\
\hline Case 2 & 309.67 & 0.0033294 & 0.00025358 \\
\hline Case 3 & 189.72 & 0.0020397 & 0.00013381 \\
\hline Case 4 & 309.67 & 0.0033294 & 0.00025358 \\
\hline
\end{tabular}
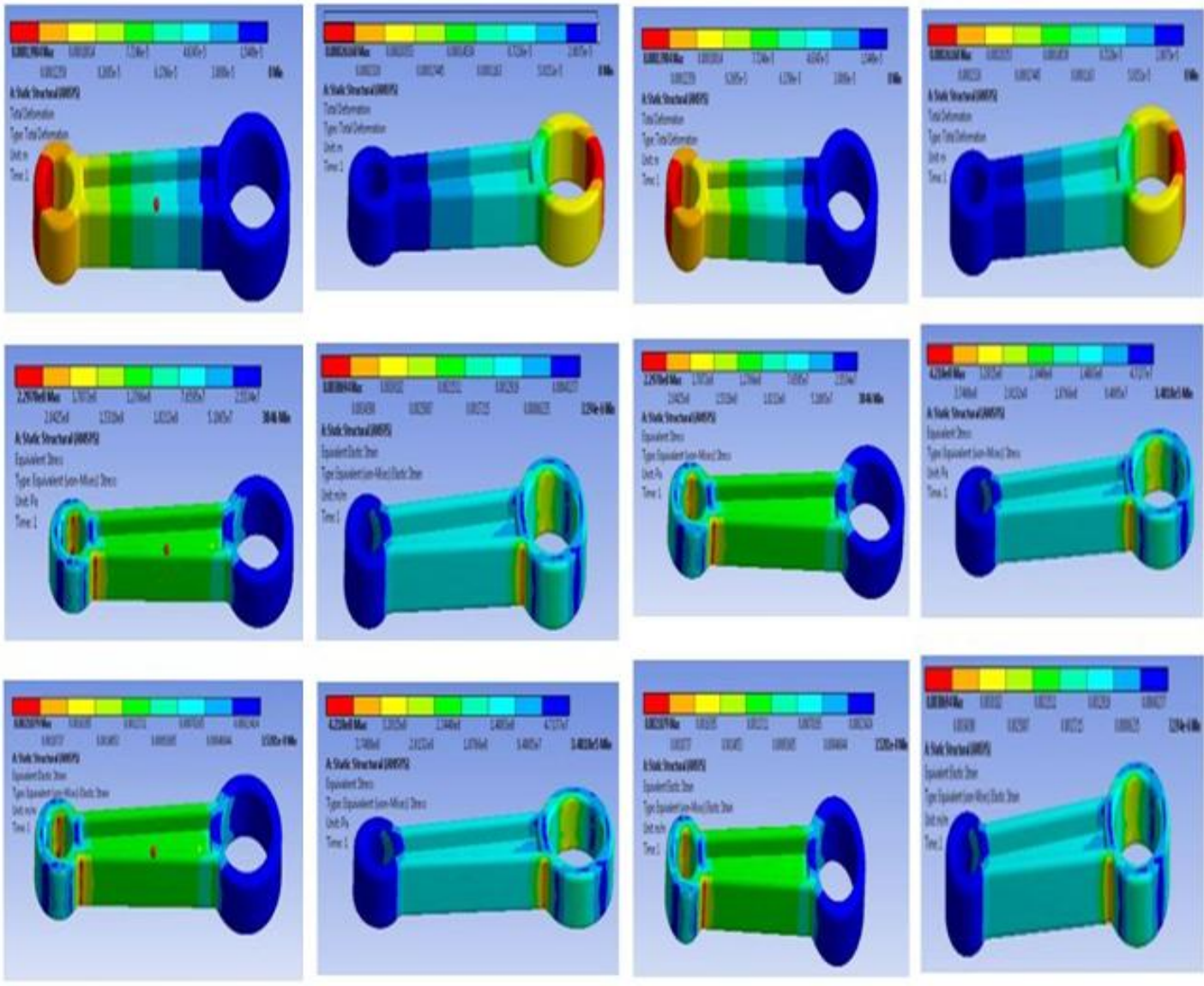

Figure 1.6: Total deformation, Equivalent Stress \& Equivalent Strain obtained for Al + 20\% SiCunderLoadingCase 1, Case 2, Case 3 and Case 4

\section{Volume 5 Issue 4, April 2016} www.ijsr.net 


\section{International Journal of Science and Research (IJSR) \\ ISSN (Online): 2319-7064}

Index Copernicus Value (2013): 6.14 | Impact Factor (2015): 6.391

Table 1.6: Combined Analysis Report for $\mathrm{Al}+20 \%$ SiC with all Loading Cases

\begin{tabular}{|l|c|c|c|}
\hline & Maximum stress $(\mathrm{MPa})$ & Maximum Strain & Total deformation $(\mathrm{m})$ \\
\hline Case 1 & 229.78 & 0.0021079 & 0.00013904 \\
\hline Case 2 & 421.88 & 0.0038964 & 0.00026168 \\
\hline Case 3 & 229.78 & 0.0021079 & 0.00013904 \\
\hline Case 4 & 421.88 & 0.0038964 & 0.00026168 \\
\hline
\end{tabular}
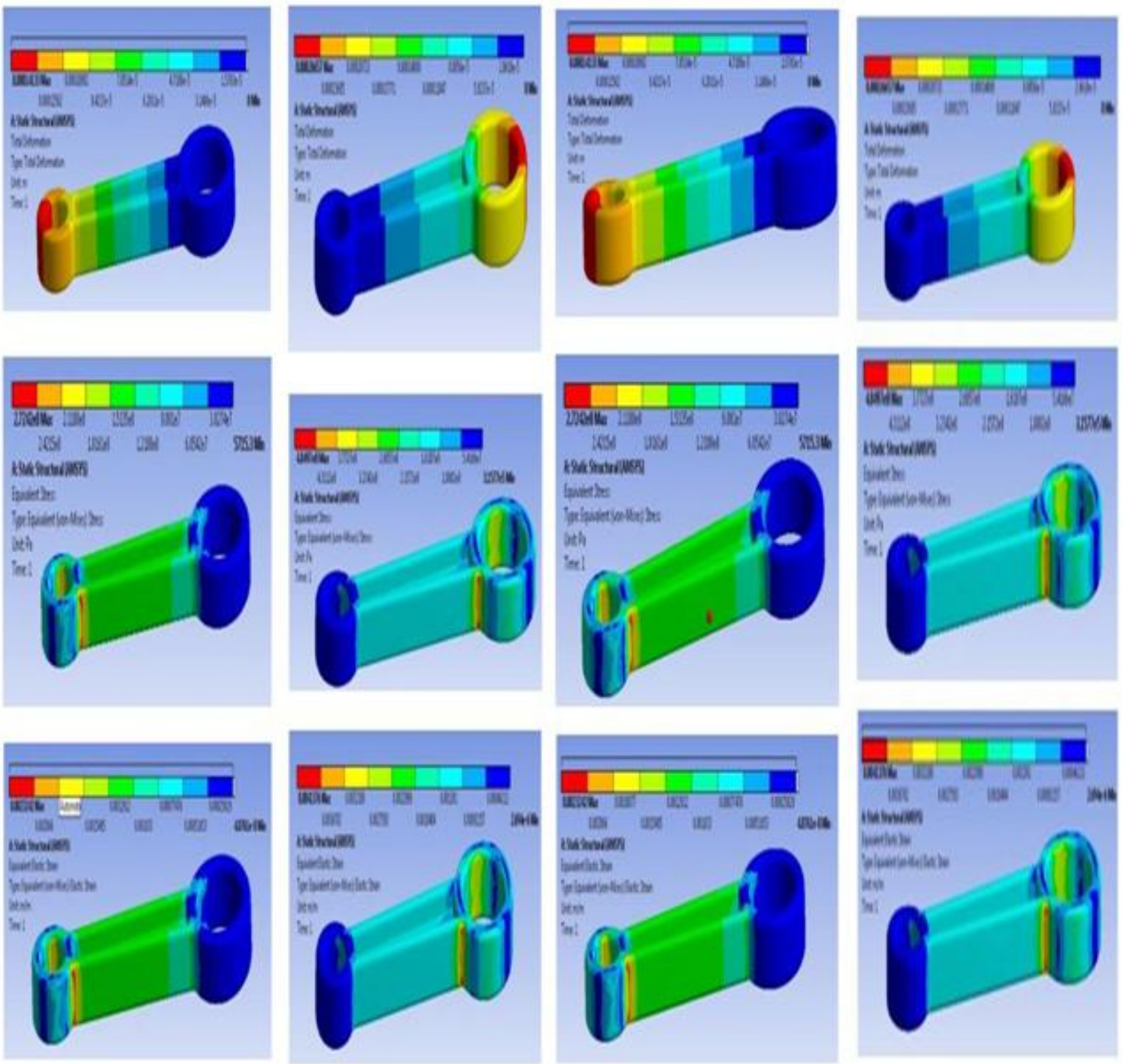

Figure 1.7: Total deformation, Equivalent Stress \& Equivalent Strain obtained for Al + 30\% SiCunderLoadingCase 1, Case 2, Case 3 and Case 4

Table 1.7: Combined Analysis Report for $\mathrm{Al}+30 \%$ SiC with all Loading Cases

\begin{tabular}{|c|c|c|c|}
\hline & $\begin{array}{c}\text { Maximum stress } \\
(\mathrm{MPa})\end{array}$ & $\begin{array}{c}\text { Maximum } \\
\text { Strain }\end{array}$ & $\begin{array}{c}\text { Total deformation } \\
(\mathrm{m})\end{array}$ \\
\hline Case 1 & 272.42 & 0.0023242 & 0.00014133 \\
\hline Case 2 & 484.97 & 0.0041376 & 0.00026657 \\
\hline Case 3 & 272.42 & 0.0023242 & 0.00014133 \\
\hline Case 4 & 484.97 & 0.0041376 & 0.00026657 \\
\hline
\end{tabular}




\section{International Journal of Science and Research (IJSR) \\ ISSN (Online): 2319-7064 \\ Index Copernicus Value (2013): 6.14 | Impact Factor (2015): 6.391}
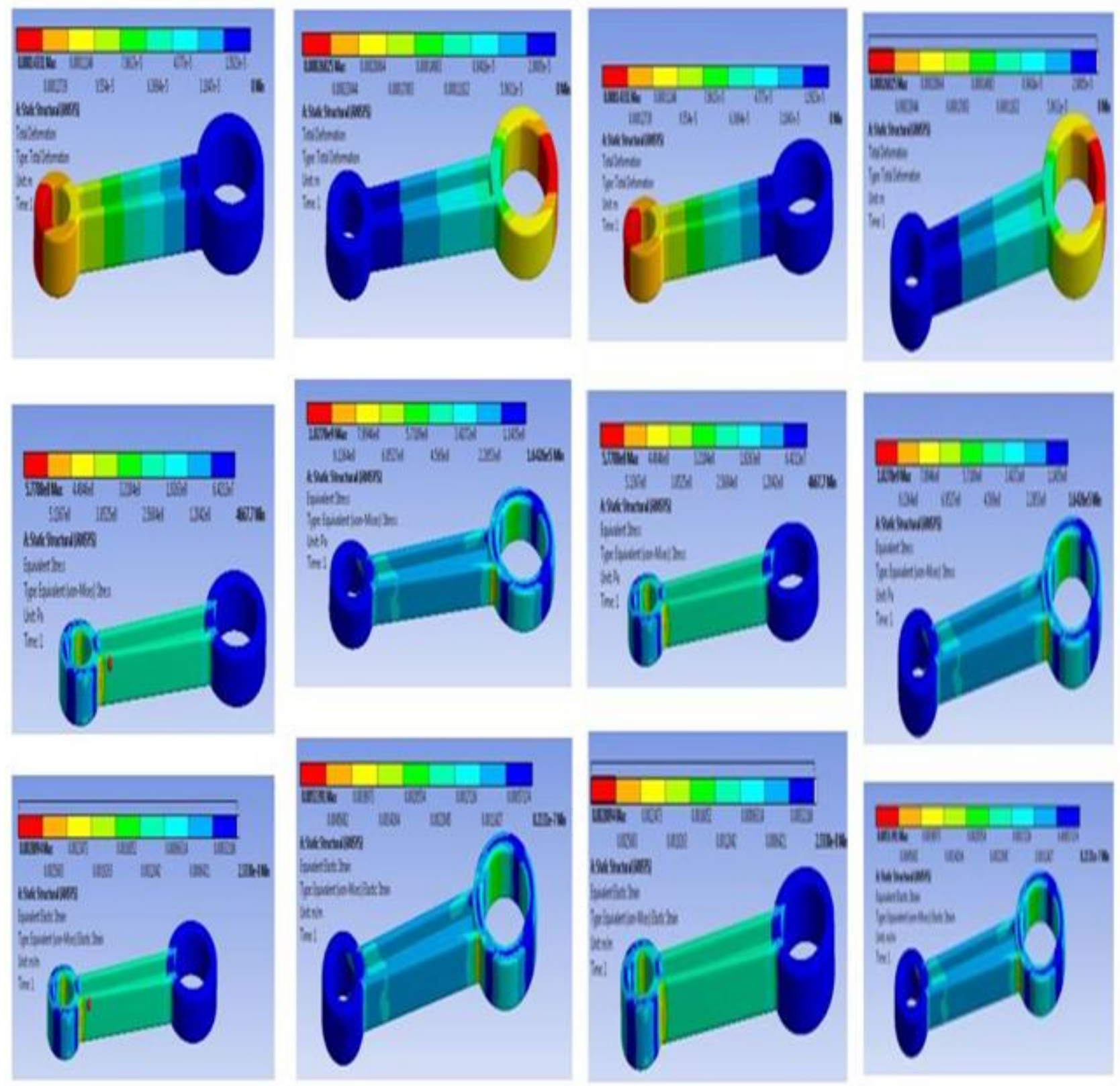

Figure 1.7: Total deformation, Equivalent Stress \& Equivalent Strain obtained for Forged Steel underLoadingCase 1, Case 2, Case 3,and Case 4

Table 1.8: Combined Analysis Report for Forged Steel with all Loading Cases

\begin{tabular}{|l|c|c|c|}
\hline & $\begin{array}{c}\text { Maximum } \\
\text { stress (MPa) }\end{array}$ & $\begin{array}{c}\text { Maximum } \\
\text { Strain }\end{array}$ & $\begin{array}{c}\text { Total } \\
\text { deformation (m) }\end{array}$ \\
\hline Case 1 & 577.88 & 0.0028894 & 0.00014331 \\
\hline Case 2 & 1027 & 0.0051391 & 0.00026825 \\
\hline Case 3 & 577.88 & 0.0028894 & 0.00014331 \\
\hline Case 4 & 1027 & 0.0051391 & 0.00026825 \\
\hline
\end{tabular}

Table 1.8: Mass of connecting rod with steel and composite materials

\begin{tabular}{|c|c|c|}
\hline \multirow{4}{*}{$\begin{array}{c}\text { Connecting } \\
\text { rod }\end{array}$} & Material Selected & weight (g) \\
\cline { 2 - 3 } & Forged steel & 230 \\
\cline { 2 - 3 } & $\mathrm{Al}+10 \% \mathrm{SiC}$ & 144 \\
\cline { 2 - 3 } & $\mathrm{Al}+20 \% \mathrm{SiC}$ & 125 \\
\cline { 2 - 3 } & $\mathrm{Al}+30 \% \mathrm{SiC}$ & 118 \\
\hline
\end{tabular}

\section{Conclusion}

In this work, exploration of connecting rod with metal matrix composite is done, aluminium is selected as matrix and various reinforcements were explored on the basis of property suitability and past usage. $\mathrm{SiC}$ is selected as suitable reinforcement with aluminium matrix for connecting rod to replace the forged steel connecting rod. Connecting rod is developed in CATIA software for dimensions obtained in designing of connecting rod as per properties of $\mathrm{Al}$ matrix and various percentage combinations of SiC. 
Table 1.9: Results Obtained

\begin{tabular}{|c|c|c|c|c|}
\hline Material & $\begin{array}{c}\text { Maximum } \\
\text { stress } \\
(\mathrm{MPa})\end{array}$ & $\begin{array}{c}\text { Maximum } \\
\text { Deformation } \\
(\mathrm{m})\end{array}$ & $\begin{array}{c}\text { Maximum } \\
\text { Strain } \\
(\mathrm{MPa})\end{array}$ & $\begin{array}{c}\text { Weight } \\
(\mathrm{g})\end{array}$ \\
\hline Forged Steel & 577.88 & 0.0001433 & 0.0028894 & 230 \\
\hline $\mathrm{Al}+10 \% \mathrm{SiC}$ & 309.67 & 0.0002536 & 0.0033294 & 144 \\
\hline $\mathrm{Al}+20 \% \mathrm{SiC}$ & 421.88 & 0.0002617 & 0.0038964 & 125 \\
\hline $\mathrm{Al}+30 \% \mathrm{SiC}$ & 484.97 & 0.0002666 & 0.0041376 & 118 \\
\hline
\end{tabular}

On observing the table $1.9, \mathrm{Al}+10 \% \mathrm{SiC}$ is selected as best composite material composition for connecting rod on the basis of maximum stress , strain, deformation and weight saving.

\section{Nomenclature}

A = cross sectional area of the connecting rod.

$\mathrm{L}=$ length of the connecting rod.

$\mathrm{C}=$ compressive yield stress.

$\mathrm{W}_{\mathrm{cr}}=$ crippling or buckling load.

Ixx $=$ moment of inertia of the section about $\mathrm{x}$-axis

Iyy $=$ moment of inertia of the section about $y$-axis

respectively.

$\mathrm{Kxx}=$ radius of gyration of the section about $\mathrm{x}$-axis

$\mathrm{W}_{\mathrm{b}}=$ Buckling load

Kyy = radius of gyration of the section about $y$ - axis

respectively.

$\mathrm{D}=$ Diameter of piston

$\mathrm{r}=$ Radius of crank

$\mathrm{n}=$ ratio of length of connecting rod

$\omega=$ Angular velocity of crank

$\mathrm{m}_{\mathrm{r}}=$ mass of reciprocating parts

$\mathrm{F}_{\mathrm{m}}=$ Maximum force

$\mathrm{F}_{\mathrm{p}}=$ Pressure force

$\mathrm{F}_{\mathrm{i}}=$ Inertia force

\section{References}

[1] Ambrish Tiwari, Jeetendra Kumar Tiwari, Sharad Kumar Chandrakar 2014. Fatigue Analysis of Connecting Rod Using Finite Element Analysis to Explore Weight and Cost Reduction Opportunities for a Production of Forged Steel Connecting Rod. International Journal of Advanced Mechanical Engineering. Volume 4. Number 7. pp. 782-802.

[2] J.D.Ramani, Prof. Sunil Shukla,Pushpendra Kumar Sharma 2014. FE-Analysis of Connecting Rod of I.C.Engine by Using Ansys for Material Optimization. Int. Journal of Engineering Research and Applications. Vol. 4. Issue 3. March 2014.Pp.216-220.

[3] Kuldeep B, Arun L.R, Mohammed Faheem, 2013. Analysis and Optimization of Connecting Rod Using Alfasic Composites. International Journal of Innovative Research in Science, Engineering and Technology. Vol. 2. Issue 6. June 2013

[4] R A Savanoor, AbhishekPatil, RakeshPatil, Amit Rodagi 2014. Finite Element Analysis of IC Engine Connecting Rod by ANSYS. International Journal of mechanical engineering \& Robotics Research, Vol. 3. No. 3. July 2014. 